\title{
Isothermal loop-mediated amplification (lamp) for diagnosis of contagious bovine pleuro-pneumonia
}

\author{
Georg Mair ${ }^{1,2}$,Edy M Vilei ${ }^{3,6}$, Abel Wade ${ }^{4}$, Joachim Frey ${ }^{3}$ and Hermann Unger $5^{5^{*}}$
}

\begin{abstract}
Background: Contagious Bovine Pleuropneumonia (CBPP) is the most important chronic pulmonary disease of cattle on the African continent causing severe economic losses. The disease, caused by infection with Mycoplasma mycoides subsp. mycoides is transmitted by animal contact and develops slowly into a chronic form preventing an early clinical diagnosis. Because available vaccines confer a low protection rate and short-lived immunity, the rapid diagnosis of infected animals combined with traditional curbing measures is seen as the best way to control the disease. While traditional labour-intensive bacteriological methods for the detection of M. mycoides subsp. mycoides have been replaced by molecular genetic techniques in the last two decades, these latter approaches require well-equipped laboratories and specialized personnel for the diagnosis. This is a handicap in areas where CBPP is endemic and early diagnosis is essential.

Results: We present a rapid, sensitive and specific diagnostic tool for M. mycoides subsp. mycoides detection based on isothermal loop-mediated amplification (LAMP) that is applicable to field conditions. The primer set developed is highly specific and sensitive enough to diagnose clinical cases without prior cultivation of the organism. The LAMP assay detects M. mycoides subsp. mycoides DNA directly from crude samples of pulmonary/ pleural fluids and serum/plasma within an hour using a simple dilution protocol. A photometric detection of LAMP products allows the real-time visualisation of the amplification curve and the application of a melting curve/re-association analysis presents a means of quality assurance based on the predetermined strand-inherent temperature profile supporting the diagnosis.
\end{abstract}

Conclusion: The CBPP LAMP developed in a robust kit format can be run on a battery-driven mobile device to rapidly detect $M$. mycoides subsp. mycoides infections from clinical or post mortem samples. The stringent innate quality control allows a conclusive on-site diagnosis of CBPP such as during farm or slaughter house inspections.

Keywords: CBPP Mycoplasma mycoides, Isothermal, Loop-mediated amplification LAMP, Molecular diagnostic, Field test

\section{Background}

Contagious Bovine Pleuropneumonia (CBPP) caused by Mycoplasma mycoides subsp. mycoides (previously further specified as Small Colony (SC) type) (Manso-Silván et al. [1]) is considered as the main plague of cattle in Africa since the eradication of rinderpest (Roeder [2]). In recent years, CBPP re-emerged from where it had persisted and has spread to

\footnotetext{
* Correspondence: h.unger@iaea.org

${ }^{5}$ Animal Production and Health Section, Joint FAO/IAEA Division of Nuclear

Techniques in Food and Agriculture, Wagramer Strasse 5, P.O. Box 100, Vienna A-1400, Austria

Full list of author information is available at the end of the article
}

areas from which it had previously been eradicated, thus stimulating new, large-scale efforts to contain or possibly eradicate epidemics (www.fao.org). The pathogen is considered highly contagious and often there is only a slow development of clinical signs. Current vaccines against CBPP confer only partial and short-lived protection and there is some concern regarding their biosafety (Mbulu et al. [3]; Thiaucourt et al. [4]; Wesonga et al. [5]). Hence, rapid and reliable diagnosis of CBPP combined with traditional control measures including reduction of animal movement and isolation or stamping-out of affected animals, remains the most efficient way to control the disease. Serological methods are generally used for determining the CBPP status at herd level 
(Amanfu et al. [6]; Bruderer et al. [7]; Le Goff and Thiaucourt [8]; Muuka et al. [9]; Schubert et al. [10]; Tardy et al. [11]). Bacterial isolation is still the reference method for detection of the pathogen. However, bacterial cultivation of mycoplasma species in general and subsequent identification is cumbersome due to the fastidious growth requirements of these bacteria.

During the last two decades, several conventional and real-time PCR methods (qPCR) for rapid diagnosis of CBPP and early outbreak warning have been developed (Bashirrudin et al. [12]; Fitzmaurice et al. [13]; Lorenzon et al. [14]; Miserez et al. [15]; Schnee et al. [16]; Vilei and Frey [17]). Although such methods are specific and sensitive they depend on a laboratory infrastructure with sophisticated equipment and highly trained personnel. Most PCR protocols for CBPP diagnosis rely on the samples being pre-enriched for mycoplasmas and/or require expensive and laborious extraction methods. Such methods are not generally available in the rural areas of Africa where CBPP is possibly endemic and rapid diagnosis of the disease is essential. Another issue is that transportation of infected samples over long distances drastically affects the viability of the bacteria rendering them unfit for culturing and/or resulting in spoilage of the nucleic acid. To address the need for an in-the-field diagnostic method for CBPP, an isothermal loop-mediated amplification (LAMP) has been developed (Notomi et al. [18]). This is based on autocycling DNA synthesis under isothermal conditions in the presence of a thermophilic strand-displacing DNA polymerase that contains the $5^{\prime} \rightarrow 3^{\prime}$ polymerase activity but lacks $5^{\prime} \rightarrow 3^{\prime}$ exonuclease activity (such as Bst or Bsm polymerase). The technique uses four specific primers recognizing six distinct regions on the DNA template (Notomi et al. [18]). The CBPP LAMP developed is fulfilling most of the criteria for an Affordable, Sensitive, Specific, User-friendly, Robust and rapid, Equipment free and Deliverable to the end user (ASSURED) diagnostic system (Mabey et al. [19]).

\section{Methods}

\section{Mycoplasma strains and DNA extraction}

In this study, 83 strains of mycoplasmas representing 17 different species or subspecies including 28 isolates of $M$. mycoides subsp. mycoides were used (Additional file 1: Table S1). Mycoplasmas were grown in a standard medium (Axcell Biotechnologies, St. Genis l'Argentière, France) at $37^{\circ} \mathrm{C}$ to a density of $10^{8}-10^{9}$ colony-forming units (CFU) per $\mathrm{ml}$ or on solid mycoplasma agar medium (Axcell Biotechnologies). Handling of live M. mycoides subsp. mycoides was performed in a biological safety laboratory fulfilling the BL3 containment safety standards. Lysis of mycoplasmas with GES buffer (5 M guanidium thiocyanate, $100 \mathrm{mM}$ EDTA, $0.5 \% \mathrm{~N}$-lauroylsarcosine) and extraction of genomic DNA were performed as previously described (Pilo et al. [20]).

\section{Clinical samples}

To allow for the use of the LAMP for diagnosis in the field, the amplification should effectively work without the need to extract DNA. This was studied using clinical samples of pleural fluid and bronchial lavage. Sequential bronchial lavage fluids were collected from cattle during an earlier experimental infection trial (Miserez et al. [15]; ethical approval by Swiss Federal Ministry of Veterinary Affairs). In addition, simulated clinical samples consisting of M. mycoides subsp. mycoides suspension supplemented with blood or squeezed liver tissues were prepared. The samples from inoculated cattle from Cameroun were from a vaccination trial (ethically approval by the board of directors of LANAVET, Cameroun; 2010).

\section{Sample preparation}

Samples $(1.5 \mu \mathrm{l})$ were added to $25 \mu \mathrm{l}$ of LAMP buffer (66 mM Tris- $\mathrm{HCl} \mathrm{pH} \mathrm{8.8,} 32 \mathrm{mM} \mathrm{KCl}, 32 \mathrm{mM}\left(\mathrm{NH}_{4}\right)_{2} \mathrm{SO}_{4}$, $16 \mathrm{mM} \mathrm{MgCl}_{2}, 0.3 \%$ (v/v) Tween 20) and heated for five minutes at $95^{\circ} \mathrm{C}$ (a process available in the reading device), followed by immediate chilling and pulse centrifugation to sediment precipitates. Chilling was necessary before addition to the LAMP master mix to avoid damaging the strand displacement polymerase.

\section{LAMP primer design}

The gene sequence encoding a putative pro-lipoprotein of M. mycoides subsp. mycoides, locus tag MSC_0500 (GenBank accession number: BX293980.2 from the type strain PG1; Westberg et al. [21]) was selected for primer design. Since the initial primer design with PrimerExplorer 3.0 (Eicken, Japan; http://primerexplorer. jp/elamp3.0.0/index.html) did not yield a performing primer set (high GC content), we first designed the outer primers Msc3.1-F3 and Msc3.1-B3 (Table 1) with Primer Express 2.0 (Life Technologies, Grand Island NY, USA) according to the heat map profile. These colder sequences were then applied to PrimerExplorer 3.0 for the design of the inner primers Msc3.1-FIP and Msc3.1-BIP (Table 1). The melting temperatures $(\mathrm{Tm})$ of the resulting primer sequences were all in the range of $56^{\circ} \mathrm{C}$. Primers were then tested for hairpin structures, extensible primer hybrids and specificity by NetPrimer (PREMIER Biosoft, USA;

\begin{tabular}{l} 
Table $\mathbf{1}$ Nucleotide sequences of the CBPP LAMP primers \\
used \\
\hline Msc3. ACTACTTGTTGTTGAGTGTTTG \\
1-F3 \\
Msc3. ATGGTGGTTTATCAAATGATGA \\
1-B3 \\
Msc3. TCAAAGAGATAATTCTACTTCAGCTAAGGATCCTGAAGAACCAGAA \\
1-FIP \\
MsC3. TCTAGCAGAATTITTGCACTTAACAAAGTTGATAACGCAATAGAGC \\
1-BIP
\end{tabular}


http://www.premierbiosoft.com/netprimer/index.html) and Primer-BLAST (http://www.ncbi.nlm.nih.gov/tools/ primer-blast/).

\section{LAMP reaction conditions}

The total volume of the LAMP assays was $25 \mu$ i.e. $15 \mu \mathrm{l}$ of LAMP buffer including the sample and $10 \mu \mathrm{l}$ of master mix for final concentrations of $460 \mathrm{mM}$ trehalose (alternative enhancer), $0.4 \mathrm{mM}$ dNTP, $1.25 \mu \mathrm{M}$ of each of the inner primers (Msc3.1-FIP and Msc3.1-BIP) and $0.25 \mu \mathrm{M}$ of each of the outer primers (Msc3.1-F3 and Msc3.1-B3). The reaction mix also contained $8 \mathrm{U}$ Bsm polymerase (Fermentas Thermo Scientific, St. Leon-Rot, Germany) and $0.4 \times$ EvaGreen $^{\circ}$ dye (20×; Biotinum Inc., Hayward, CA, USA). Standard reactions were run for $60 \mathrm{~min}$ at $58^{\circ} \mathrm{C}$ in an ESEQuant Tube Scanner TS95 (Qiagen, Hilden, Germany) recording fluorescence from DNA bound EvaGreen once per minute. The optimal reaction temperature was predetermined on a gradient real-time cycler (StepOnePlus ${ }^{\mathrm{Tm}}$; Life Technologies, Grand Island NY, US).

\section{Quality assurance}

To allow for a quality assurance protocol, the standard procedure for real-time PCR, melting curve analysis (MCA) and an alternatively re-association curve analysis was tested on a real-time platform (Rotor-Gene Q, Qiagen, Hilden Germany). After the LAMP process, for MCA samples were heated from $65^{\circ} \mathrm{C}$ to $90^{\circ} \mathrm{C}$ in $1^{\circ} \mathrm{C} /$ min steps with consecutive measurement at each step. For re-association of melted DNA (amplicons), reactions were cooled down from $90^{\circ} \mathrm{C}$ to $65^{\circ} \mathrm{C}$ in the same fashion. The amplicon inherent Tm was assessed with Oligo Calc (http://www.basic.northwestern.edu/biotools/oligocalc.html) and POLAND (http://www.biophys.uni-duesseldorf.de/ html/local/POLAND/poland.html).

\section{LAMP result evaluation}

LAMP is a continuous process, unlike PCR, with parallel amplification and strand separation. Thus, only a "quasi" exponential function can be expected in short time increments and only the measurement of fluorescence in intervals of 1 minute or longer presents a continuous sigmoidal curve. For this exercise, we chose $1 \mathrm{~min}$ increments to evaluate the curve and defined an increase of $>30 \mathrm{mV} /$ minute as a significant increase (Additional file 1). This algorithm was implemented in the Tube Scanner software and has the following limiting factors: the baseline starts after the third measurement and ends after the seventh, whereas the mean of the $\mathrm{mV}$ values represents the baseline value (Bv). The curve is classified as positive if the increase of signal (slope) above the mean of the baseline is $>30 \mathrm{mV}$ for a minimum of 3 measurements (Additional file 1). The absolute time-point of detection $\mathrm{Td}$ (quantification) is determined when the slope increase is $>30 \mathrm{mV}$ for the first time at a tube scanner excitation intensity of $36 \%$.

\section{Results}

Evaluation of analytical sensitivity and specificity

To evaluate the specificity of the CBPP LAMP reaction, templates of purified DNA samples corresponding to approximately $10^{6}$ genome equivalents or suspensions of mycoplasmal cultures corresponding to approximately $10^{6}$ cells from 28 different strains of $M$. mycoides subsp. mycoides and from 57 strains of 15 other related mycoplasma species or subspecies (Additional file 1: Table S1) were examined in the CBPP LAMP. The CBPP LAMP gave positive signals for all 28 strains of $M$. mycoides subsp. mycoides, but not for any of the other 57 mycoplasma species. The LAMP reactions for the negative control remained negative, even when the reactions extended to 90 minutes.

To assess the limit of detection, a series of 10 fold dilutions of a suspension culture of $M$. mycoides subsp. mycoides strain Afadé were used as template. As shown in Figure 1, the method detects 1000 cells per reaction at a Time of detection ( $\mathrm{Td}$ ) of 45 minutes, while the detection of 10 cells requires an extended analysis time of $\mathrm{Td}=67$ minutes.

\section{Quality assurance}

For pathogen diagnosis, a quality assurance protocol is necessary. We performed MCA, the standard for qPCR and compared the result with the Tm for our CBPP amplicon calculated using two methods (nearest neighbour and Poland algorithm, respectively; Breslauer et al. [22]; Steger [23]). Interestingly, the calculated $\operatorname{Tm}\left(74.9^{\circ} \mathrm{C}\right)$ did not correspond to the values measured $\left(78.4\right.$ to $79.0^{\circ} \mathrm{C}$, dependent on the sample origin) (Figure 2). The analysis of the inverse protocol, re-association curve analysis (RCA)

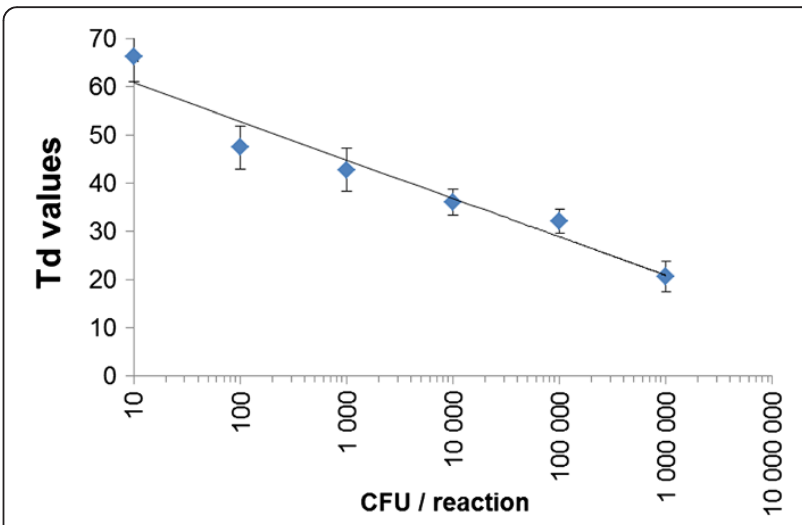

Figure 1 Sensitivity of the CBPP LAMP: Titration curve based on diluted culture samples of $M$. mycoides subsp. mycoides strain Afadé directly employed in the reaction. 


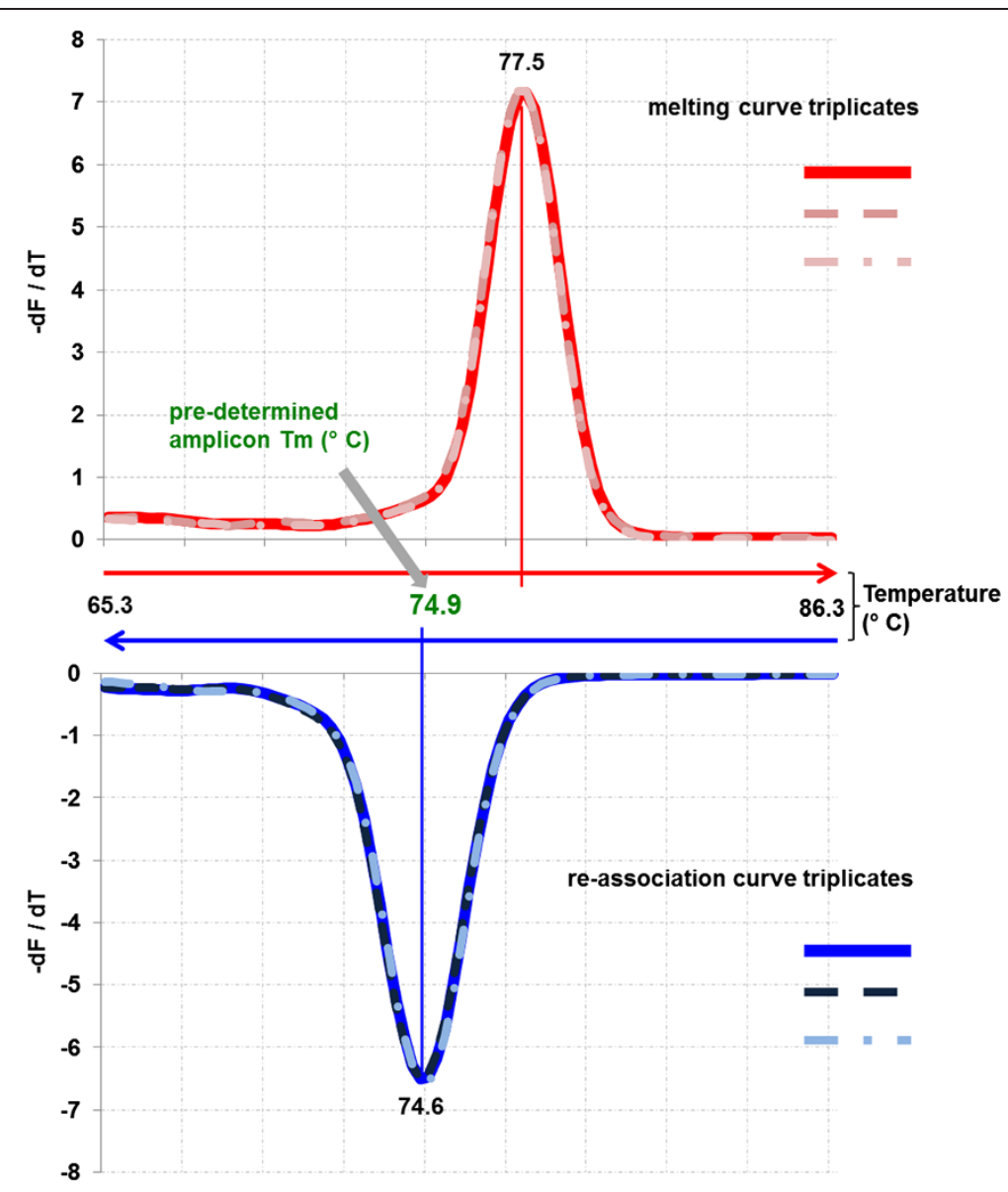

Figure 2 Melting curve and re-association curve analysis of $M$. mycoides subsp. mycoides type strain PG1; directly from broth culture. The pre-determined $\operatorname{Tm}\left(74.9^{\circ} \mathrm{C}\right)$ corresponds to the value achieved through re-association $\left(74.6^{\circ} \mathrm{C}\right)$ of exo-thermally separated LAMP products.

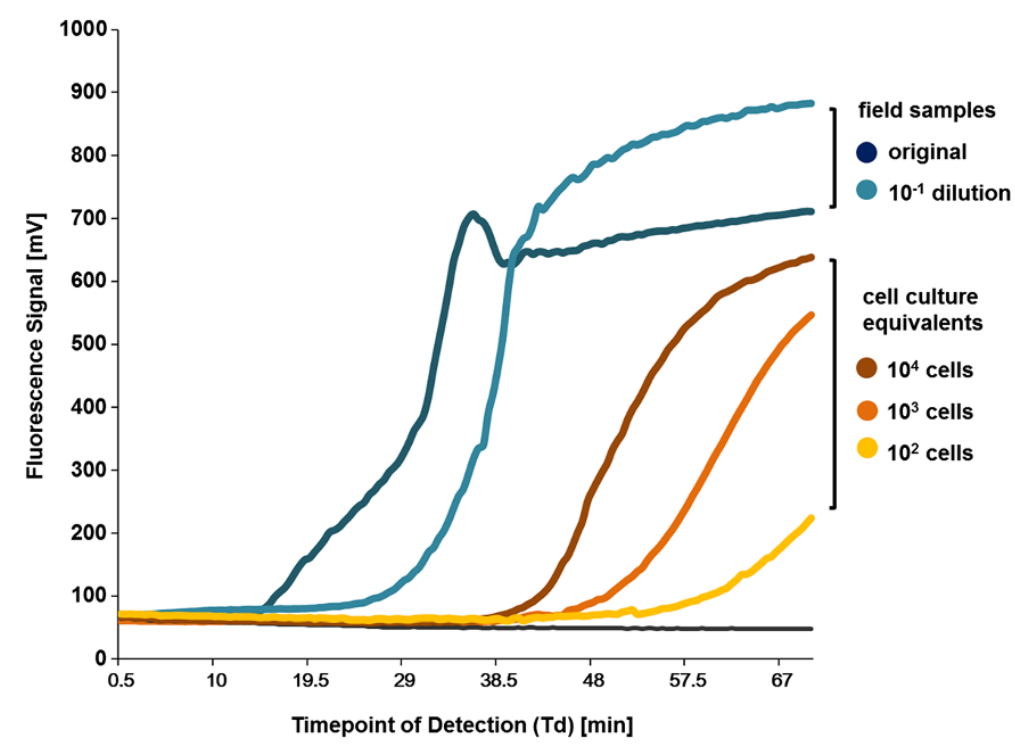

Figure 3 Calibration of the CBPP LAMP. Upper panel: Fluorometric output of LAMP amplifications using non-processed pleural fluid samples from a CBPP infected cow. Lower panel: Titration of M. mycoides subsp. mycoides strain Afadé culture fluid at various concentrations. 
gave values matching the calculated ones thus allowing a reliable QA for CBPP LAMP diagnosis.

\section{Analysis of clinical samples}

The assessment of the test performance for the crude samples gave for the spiked samples a $\mathrm{Td}$ of around 45 minutes $\left(10^{3} \mathrm{Mmm} /\right.$ reaction). Pleural fluid samples from two cows, with bacteriologically confirmed CBPP taken at necropsy, resulted in $\mathrm{Td}$ values ranging between 20 and 35 minutes (Figure 3).

LAMP analyses of bronchial lavage samples taken sequentially from in-contact animals (Miserez et al. [15]) gave very similar results to the highly sensitive nested PCR based on P72 gene encoding the lipoprotein LppA (Tables 2, 3), showing that the CBPP LAMP was suitable for both clinical samples from necropsy and from live animals. The AFADE 16 samples gave identical results. The LAMP was additionally positive 4 times and the

Table 2 Detection of $M$. mycoides subsp. mycoides in bronchial lavage fluid of cattle that were experimentally infected by contact with animals that were directly infected 4 weeks before with $M$. mycoides subsp. mycoides a) strain Afadé [15] or b) strain L2 [15]

\begin{tabular}{|c|c|c|c|}
\hline $\begin{array}{l}\text { Days before/after } \\
\text { contact infection }\end{array}$ & $\begin{array}{l}\text { Viable } M . \text { mycoides } \\
\text { subsp. mycoides / ml }\end{array}$ & Nested PCR* & LAMP \\
\hline-28 & & - & - \\
\hline-7 & & - & - \\
\hline+13 & $1.3 \times 10^{4}$ & + & + \\
\hline+21 & $4.0 \times 10^{4}$ & + & + \\
\hline+28 & $3.5 \times 10^{3}$ & + & + \\
\hline+35 & $2.5 \times 10^{3}$ & + & + \\
\hline+42 & $7.0 \times 10^{7}$ & + & + \\
\hline+49 & $5.5 \times 10^{6}$ & + & + \\
\hline+56 & $1.8 \times 10^{7}$ & + & + \\
\hline+63 & $7.0 \times 10^{8}$ & + & + \\
\hline+70 & & - & + \\
\hline+77 & & - & - \\
\hline+84 & & - & - \\
\hline+92 & & - & + \\
\hline+98 & & - & - \\
\hline+105 & $1.0 \times 10^{2}$ & + & + \\
\hline+112 & & - & + \\
\hline+119 & & - & + \\
\hline+126 & & + & - \\
\hline+134 & & - & + \\
\hline+141 & & - & - \\
\hline+147 & & - & - \\
\hline
\end{tabular}

* nested PCR results [15].
Table 3 Detection of $\boldsymbol{M}$. mycoides subsp. mycoides in bronchial lavage fluid of cattle that were experimentally infected by contact with animals that were directly infected 4 weeks before with $M$. mycoides subsp. mycoides a) strain Afadé or b) strain L2 (Miserez et al., [15])

\begin{tabular}{|c|c|c|c|}
\hline $\begin{array}{l}\text { Days before/after } \\
\text { contact infection }\end{array}$ & $\begin{array}{l}\text { Viable } M . \text { mycoides } \\
\text { subsp. mycoides / ml }\end{array}$ & Nested PCR* & LAMP \\
\hline-21 & & - & - \\
\hline+16 & & - & - \\
\hline+21 & & - & - \\
\hline+29 & & - & - \\
\hline+35 & & - & - \\
\hline+42 & & - & - \\
\hline+49 & & - & - \\
\hline+56 & & - & - \\
\hline+64 & & - & - \\
\hline+70 & & - & + \\
\hline+77 & $1.0 \times 10^{2}$ & + & + \\
\hline+84 & & + & + \\
\hline+92 & $2.5 \times 10^{2}$ & + & + \\
\hline+100 & $3.5 \times 10^{2}$ & + & + \\
\hline+105 & $8.5 \times 10^{2}$ & + & + \\
\hline+112 & $1.0 \times 10^{2}$ & + & + \\
\hline+119 & $1.0 \times 10^{2}$ & + & + \\
\hline+125 & $1.0 \times 10^{2}$ & + & + \\
\hline+132 & & + & + \\
\hline+140 & & - & + \\
\hline+146 & & - & + \\
\hline+153 & & + & + \\
\hline+160 & & + & + \\
\hline+168 & & - & + \\
\hline+175 & & + & + \\
\hline+181 & & + & + \\
\hline+188 & $1.0 \times 10^{2}$ & + & + \\
\hline+195 & $1.0 \times 10^{2}$ & + & + \\
\hline+202 & & - & - \\
\hline+209 & & - & - \\
\hline+216 & & - & + \\
\hline+223 & & - & - \\
\hline
\end{tabular}

* nested PCR results [15].

n-PCR was positive once more. For the L2 strain, 27 samples corresponded and additionally 5 times only LAMP was positive.

Samples collected from a challenge trial in Cameroun revealed that not only were pulmonary samples and pleural fluids suitable for $\mathrm{Mmm}$ detection by LAMP, but the serum also gave valid results (Table 4). 


\begin{tabular}{|c|c|c|c|c|c|}
\hline Origin & Specimen ID & PCR & cELISA & Culture & LAMP \\
\hline \multirow[t]{2}{*}{ Mmm inoculated cow, 8429} & Serum & nd & + & nd & + \\
\hline & Pleural fluid & + & nd & + & + \\
\hline \multirow[t]{2}{*}{ Mmm inoculated cow, 8462} & Serum & nd & + & nd & + \\
\hline & Pleural fluid & + & nd & + & + \\
\hline \multirow[t]{3}{*}{ Mmm inoculated cow, 05} & Serum & nd & + & nd & + \\
\hline & Lung & + & nd & + & + \\
\hline & Pleural fluid & + & nd & + & + \\
\hline Bov. before inoculation; 8178 & Serum & - & nd & nd & - \\
\hline Mmm inoculated cow, 8178 & Serum & nd & + & nd & + \\
\hline \multirow[t]{2}{*}{ Mmm inoculated cow, 07} & Lung & + & nd & + & + \\
\hline & Pleural fluid & + & nd & + & + \\
\hline \multirow[t]{2}{*}{ Mmm inoculated cow, 08} & Lung & + & nd & + & + \\
\hline & Pleural fluid & + & nd & + & + \\
\hline \multirow[t]{2}{*}{ Mmm inoculated cow, 8454} & Serum & nd & + & nd & + \\
\hline & Serum & nd & + & nd & + \\
\hline Mmm inoculated cow, 49 & Lung & + & nd & + & + \\
\hline Mmm inoculated cow, 50 & Lung & + & nd & + & + \\
\hline Mmm inoculated cow, 56 & Pleural fluid & + & nd & + & + \\
\hline \multirow[t]{2}{*}{ Mmm inoculated cow, 8410} & Lung & - & nd & nd & - \\
\hline & Pulmon. lymph node & - & nd & nd & - \\
\hline
\end{tabular}

nd: not determined.

\section{Discussion and conclusion}

Sensitive, reliable and time-saving molecular diagnostic tests, vital for the successful control of CBPP, mostly rely on TaqMan-based real-time PCR for the detection of the pathogen M. mycoides subsp. mycoides in extracted and often pre-cultured clinical samples (Gorton et al. [24]; Schnee et al. [16]; Vilei and Frey [17]). Whilst these are broadly accepted as the most specific and sensitive method and as reference tests in control and surveillance programs, they depend on an elaborate and expensive laboratory infrastructure; sophisticated equipment and personnel with advanced molecular diagnostic knowledge hence, are not applicable to field conditions where CBPP is endemic and where on-site diagnosis is vital to provide confirmation of disease. A new and highly efficient isothermal amplification protocol for the detection of CBPP was developed that offers the same advantages of specificity and sensitivity of the real-time PCR methodology, but does not need the sophisticated laboratory-based equipment and is thus applicable to field conditions. This was accomplished through the design of a specific LAMP primer set for an already optimized master mix. To make the test suitable for the field, the master and the primer mix were lyophilized and are thus thermostable for a longer period ( $>1$ year) and when rehydrated for at least one week on the bench. Additionally, the ready mixes allow the test to be carried out in only two pipetting steps (sample dilution and master mix assembly) thus avoiding errors and reducing training needs of operators.

Because the sample preparation and the amplification steps are run in a battery-driven instrument, the test can easily be performed at any location. The continuous reading of the fluorescence during amplification allows a real-time analysis of the results by the introduction of a minimum absolute increase value for positivity thus avoiding background problems usually faced with threshold values. The interpretation of results simply consists of monitoring the fluorescence increase of more than $30 \mathrm{mV}$ for a minimum of two consecutive readings, which corresponds to a positive sample. Furthermore, these evaluation parameters can be programmed and therefore only the results are displayed on the instrument.

A QA procedure was established corresponding to the published Tm prediction models (Steger [23]; Schütz and van Ahsen [25]; Kibbe [26]). Despite the wide application of melting curve analysis (MCA), the re-association analysis in our setup gives the advantage of a direct correspondence to the established prediction calculations (Breslauer et al. [22]; 
Steger [23]) and does not require integration of surrogate parameters such as enhancers or dye factors. To perform the re-association analysis, specific software for the tube scanner (ESEmelt) was developed together with the producer based on the Savitzky-Golay filter algorithm (Savitzky and Golay [27]). False positive results due to random amplification of non-specific target DNA or contamination were excluded through the re-association analysis carried directly after amplification step without opening the vessel.

The analytical sensitivity (titration) of our test was comparable to the diagnostic sensitivity (clinical samples) better than, the real-time PCR or nested PCR. As in real-time PCR, heat-inactivated samples, after amplification, are kept in the closed tubes to avoid cross-contamination and to facilitate further analysis in a laboratory.

Regarding the ASSURED parameters published by Mabey the test fulfils all criteria with the exception of freedom from instrumentation. We believe that for a quality assured field result quantitative parameters which can be transmitted instantly to a reference laboratory are important thus allowing an immediate response which might be rather expensive.

The developed CBPP LAMP is an ideal, simple, robust and precise method to diagnose $M$. mycoides subsp. mycoides in an outbreak situation or during regular slaughterhouse inspections in monitoring programs for CBPP prevalence assessment.

\section{Additional file}

Additional file 1: Supplement.

\section{Competing interests}

The authors declare that they have no competing interests.

\section{Authors' contributions}

$\mathrm{HU}$ and GM contributed equally to the study design and in drafting the manuscript. GM took the lead on the assay development and initial testing. JF designed the evaluation procedure and EMV performed the evaluation of the test performance. AW carried out the field evaluation. GM and HU developed the result evaluation. JF and EMV provided critical feedback on the manuscript. All authors read and approved the final manuscript.

\section{Acknowledgements}

We would like to thank the staff of LANAVET for their help in collection of the samples. A. Wade was supported by an IAEA CRP grant (CMR 13843) and G. Mair by an IAEA technical contract (AUS 15985).

\section{Author details}

${ }^{1}$ Genomics Unit VetCore, University of Veterinary Medicine, Vienna, Austria. ${ }^{2}$ Tropical Vet. Med. Laboratory, University of Veterinary Medicine, Vienna, Austria. ${ }^{3}$ Institute of Veterinary Bacteriology, Vetsuisse, University of Bern, Bern, Switzerland. ${ }^{4}$ LANAVET, Garoua, Cameroun. ${ }^{5}$ Animal Production and Health Section, Joint FAO/IAEA Division of Nuclear Techniques in Food and Agriculture, Wagramer Strasse 5, P.O. Box 100, Vienna A-1400, Austria. ${ }^{6}$ Current address: Suisselab AG, Schützenstrasse 10, Zollikofen, BE CH-3052, Switzerland.
Received: 21 January 2013 Accepted: 14 May 2013

Published: 27 May 2013

\section{References}

1. Manso-Silván L, Vilei EM, Sachse K, Djordjevic SP, Thiaucourt F, Frey J: Mycoplasma leachii sp. nov. as a new species designation for Mycoplasma sp. bovine group 7 of Leach, and reclassification of Mycoplasma mycoides subsp. mycoides LC as a serovar of Mycoplasma mycoides subsp. capri. Int J Syst Evol Microbiol 2009, 59:1353-1358.

2. Roeder PL: Rinderpest: The end of cattle plague. Prev Vet Med 2011, 102:98-106

3. Mbulu RS, Tjipura-Zaire G, Lelli R, Frey J, Pilo P, Vilei EM, Mettler F, Nicholas RA, Huebschle OJ: Contagious bovine pleuropneumonia (CBPP) caused by vaccine strain T1/44 of Mycoplasma mycoides subsp. mycoides SC. Vet Microbiol 2004, 98:229-234.

4. Thiaucourt F, Aboubakar Y, Wesonga H, Manso-Silván L, Blanchard A: Contagious bovine pleuropneumonia vaccines and control strategies: recent data. Dev Biol (Basel) 2004, 119:99-111.

5. Wesonga $H$, Manso-Silván $L$, Thiaucourt F: CBPP vaccine strain T1/44: possible reversion to virulence. Proceedings of the $3^{\text {rd }}$ Meeting of the FAO/OIE/ OAU-IBAR/IAEA Consultative Group in CBPP in Africa. Rome. Rome: FAO Publications Rome; 2003.

6. Amanfu W, Sediadie S, Masupu KV, Raborokgwe MV, Benkirane A, Geiger R Thiaucourt F: Comparison between c-ELISA and CFT in detecting antibodies to Mycoplasma mycoides mycoides biotype SC in cattle affected by CBPP in Botswana. Ann N Y Acad Sci 2000, 916:364-369.

7. Bruderer U, Regalla J, Abdo E-M, Huebschle OJB, Frey J: Serodiagnosis and monitoring of contagious bovine pleuropneumonia (CBPP) with an indirect ELISA based on the specific lipoprotein LppQ of Mycoplasma mycoides subsp. mycoides SC. Vet Microbiol 2002, 84:195-205.

8. Le Goff C, Thiaucourt F: A competitive ELISA for the specific diagnosis of contagious bovine pleuropneumonia (CBPP). Vet Microbiol 1998, 60:179-191.

9. Muuka G, Hang'ombe BM, Nalubamba KS, Kabilika S, Mwambazi L, Muma JB: Comparison of complement fixation test, competitive ELISA and LppQ ELISA with post-mortem findings in the diagnosis of contagious bovine pleuropneumonia (CBPP). Trop Anim Health Prod 2011, 43:1057-1062.

10. Schubert E, Sachse K, Jores J, Heller M: Serological testing of cattle experimentally infected with Mycoplasma mycoides subsp. mycoides small colony using four different tests reveals a variety of seroconversion patterns. BMC Vet Res 2011, 7:72.

11. Tardy F, Gaurivaud P, Manso-Silván L, Thiaucourt F, Pellet MP, Mercier P, Le Grand D, Poumarat F: Extended surveillance for CBPP in a free country: Challenges and solutions regarding the potential caprine reservoir. Prev Vet Med 2011, 101:89-95.

12. Bashiruddin JB, Frey J, Königsson MH, Johansson KE, Hotzel H, Diller R, De Santis P, Botelho A, Ayling RD, Nicholas RA, Thiaucourt F, Sachse K: Evaluation of PCR systems for the identification and differentiation of Mycoplasma agalactiae and Mycoplasma bovis: a collaborative trial. Vet J 2005, 169:268-275.

13. Fitzmaurice J, Sewell M, Manso-Silván L, Thiaucourt F, McDonald WL, O’Keefe $J S$ : Real-time polymerase chain reaction assays for the detection of members of the Mycoplasma mycoides cluster. N Z Vet J 2008, 56:40-47.

14. Lorenzon S, Manso-Silván L, Thiaucourt F: Specific real-time PCR assays for the detection and quantification of Mycoplasma mycoides subsp. mycoides SC and Mycoplasma capricolum subsp. capripneumoniae. Mol. Cell. Probes 2008, 22:324-328.

15. Miserez R, Pilloud T, Cheng X, Nicolet J, Griot C, Frey J: Development of a sensitive nested PCR method for the specific detection of Mycoplasma mycoides subsp. mycoides SC. Mol Cell Probes 1997, 11:103-111.

16. Schnee C, Heller M, Heller M, Jores J, Tomaso H, Neubauer H: Assessment of a novel multiplex real-time PCR assay for the detection of the CBPP agent Mycoplasma mycoides subsp. mycoides SC through experimental infection in cattle. BMC Vet Res 2011, 7:47.

17. Vilei EM, Frey J: Detection of Mycoplasma mycoides subsp. mycoides SC in bronchoalveolar lavage fluids of cows based on a TaqMan real-time PCR discriminating wild type strains from an $1 \mathrm{ppQ}^{-}$mutant vaccine strain used for DIVA-strategies. J Microbiol Methods 2010, 81:211-218.

18. Notomi T, Okayama H, Masubuchi H, Yonekawa T, Watanabe K, Amino N, Hase T: Loop-mediated isothermal amplification of DNA. Nucl Acids Res 2000, 28:E63. 
19. Mabey D, Peeling RW, Ustianowski A, Perkins MD: Diagnostics for the developing world. Nat Rev Microbiol 2004, 2(3):231.

20. Pilo P, Vilei EM, Peterhans E, Bonvin-Klotz L, Stoffel MH, Dobbelaere D, Frey $\mathrm{J}$ : A metabolic enzyme as a primary virulence factor of Mycoplasma mycoides subsp. mycoides small colony. J Bacteriol 2005, 187:6824-6831.

21. Westberg J, Persson A, Holmberg A, Goesmann A, Lundeberg J, Johansson KE, Pettersson B, Uhlen M: The genome sequence of Mycoplasma mycoides subsp. mycoides $\mathrm{SC}$ type strain $\mathrm{PG} 1^{\top}$, the causative agent of contagious bovine pleuropneumonia (CBPP). Genome Res 2004, 14:221-227.

22. Breslauer $\mathrm{K}$, Frank R, Marky LA BH: Predicting DNA duplex stability from the base sequence. Proc Natl Acad Sci USA 1986, 83(11):3746-3750.

23. Steger G: Thermal denaturation of double-stranded nucleic acids: prediction of temperatures critical for gradient gel electrophoresis and polymerase chain reaction. Nucleic Acids Res 1994, 22(14):2760-2768.

24. Gorton TS, Barnett MM, Gull T, French RA, Lu Z, Kutish GF, Adams LG, Geary SJ: Development of real-time diagnostic assays specific for Mycoplasma mycoides subspecies mycoides Small Colony. Vet Microbiol 2005, 111:51-58

25. Schütz $\mathrm{E}$, Von Ahsen N: Spreadsheet software for thermodynamic melting point prediction of oligonucleotide hybridization with and without mismatches. Biotechniques 1999, 27(6):1218-1224.

26. Kibbe WA: OligoCalc: an online oligonucleotide properties calculator. Nucleic Acids Res 2007, 35(Web Server Issue):43-46.

27. Savitzky A, Golay MJE: Smoothing and differentiation of data by simplified least squares procedures. In analytical chemistry. 1964:S. 1627-S. 1639. 36, Nr. 8, 1. Juni.

doi:10.1186/1746-6148-9-108

Cite this article as: Mair et al:: Isothermal loop-mediated amplification (lamp) for diagnosis of contagious bovine pleuro-pneumonia. BMC Veterinary Research 2013 9:108.

\section{Submit your next manuscript to BioMed Central and take full advantage of:}

- Convenient online submission

- Thorough peer review

- No space constraints or color figure charges

- Immediate publication on acceptance

- Inclusion in PubMed, CAS, Scopus and Google Scholar

- Research which is freely available for redistribution 